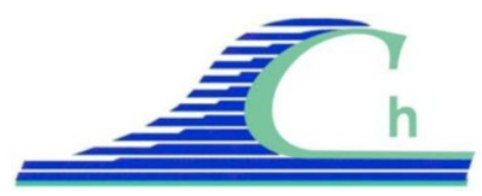

XII ${ }^{\text {èmes }}$ Journées Nationales Génie Côtier - Génie Civil

Cherbourg, 12-14 juin 2012

DOI:10.5150/jngcgc.2012.020-B @ Editions Paralia CFL

disponible en ligne - http://www.paralia.fr - available online

\title{
Ressaut de marée et mascaret - exemples de la Garonne et de la Seine
}

\author{
Natalie BONNETON ${ }^{1}$, Philippe BONNETON ${ }^{1}$, \\ Jean-Paul PARISOT ${ }^{1}$, Aldo SOTTOLICHIO ${ }^{1}$
}

\author{
1. Université Bordeaux 1, CNRS, UMR 5805-EPOC, Talence, F-33405 France. \\ n.bonneton@epoc.u-bordeaux1.fr
}

\section{Résumé :}

Nous présentons dans cet article des résultats expérimentaux nouveaux sur les ressauts de marée se propageant dans la Garonne et la Seine. Nous avons mis en évidence que lorsque le nombre de Froude dépasse une valeur critique $\mathrm{F}_{\mathrm{c}}$ il y a une forte amplification de la cambrure des ondes secondaires associées au ressaut. Ce régime d'onde de "forte cambrure" correspond au phénomène qu'on dénomme couramment mascaret. Nous avons montré que les ressauts de marée, incluant le régime à "faible cambrure" $\left(\mathrm{F}<\mathrm{F}_{\mathrm{c}}\right)$, étaient présents dans la Garonne pour une large majorité de marées et pouvaient même être observés par morte eau. Nous avons aussi identifié des ressauts de marée de "faible cambrure" dans la Seine et présenté les premières mesures quantitatives de ce phénomène dont la hauteur de la première vague peut dépasser $1 \mathrm{~m}$.

\section{Mots-clés :}

Estuaire - Fleuve - Onde de marée - Hydraulique - Non-hydrostatique - Non-linéaire Déferlement - Transport sédimentaire - Ecosystème estuarien

\section{Abstract:}

In this paper we present new experimental results on tidal bores propagating in the Garonne and the Seine rivers. We have shown that the secondary wave steepness strongly increase when the Froude number exceeds a critical value $\mathrm{F}_{\mathrm{c}}$. The largesteepness tidal bore regime corresponds to what is commonly termed in French mascaret. We have shown that in the Garonne River tidal bores occur for a large majority of tides, even sometimes at neap tides. We have also identified small-steepness tidal bores in the Seine River and presented the first quantitative measurements of this phenomenon.

\section{Keywords:}

Estuary - River - Tidal wave - Hydraulic - Non-hydrostatic - Nonlinear - Breaking Sediment transport - Estuarine ecosystem

\section{Introduction}

Lorsqu'une marée de forte amplitude se propage dans un estuaire convergent de faible pente on observe fréquemment, au début du flot, la formation et la propagation d'un ressaut en translation. Nous dénommerons par la suite ce phénomène : ressaut de marée 
(terminologie inspirée de "tidal bore" en anglais). Ce phénomène a été très peu étudié en milieu naturel (ex.: SIMPSON et al., 2004; WOLANSKI et al., 2004). Nous présentons dans cet article les campagnes de mesures réalisées en 2010 et 2011 pour la caractérisation des ressauts de marée (dénommés $R M$ dans la suite de l'article) dans la Garonne et la Seine. Nous présentons la première analyse quantitative de l'occurrence des $R M$ dans la Garonne, ainsi que les premières mesures des $R M$ ondulés dans la Seine. Dans des canaux rectangulaires pour le régime non-déferlant il a été observé depuis longtemps (FAVRE, 1935) que la structure des ondes secondaires évolue progressivement lorsque le nombre de Froude augmente, avec en particulier une augmentation de l'amplitude et une diminution de la longueur d'onde. Nous montrerons que dans un milieu fluvial naturel, avec des berges en pente douce, il existe un changement de régime pour les $R M$ oscillants autour d'un nombre de Froude critique $\mathrm{F}_{\mathrm{c}}$. Pour $\mathrm{F}>\mathrm{F}_{\mathrm{c}}$, on observe des ondes secondaires de fortes cambrures aux caractéristiques voisines de celles observées en canal rectangulaire. Ce type de $R M$, énergétique et visuellement observable, correspond au phénomène dénommé en Français mascaret ou la barre. En revanche, pour $\mathrm{F}<\mathrm{F}_{\mathrm{c}}$ les $\mathrm{RM}$ sont caractérisés par une cambrure beaucoup plus faible que celle observée en canal rectangulaire pour un même nombre de Froude.

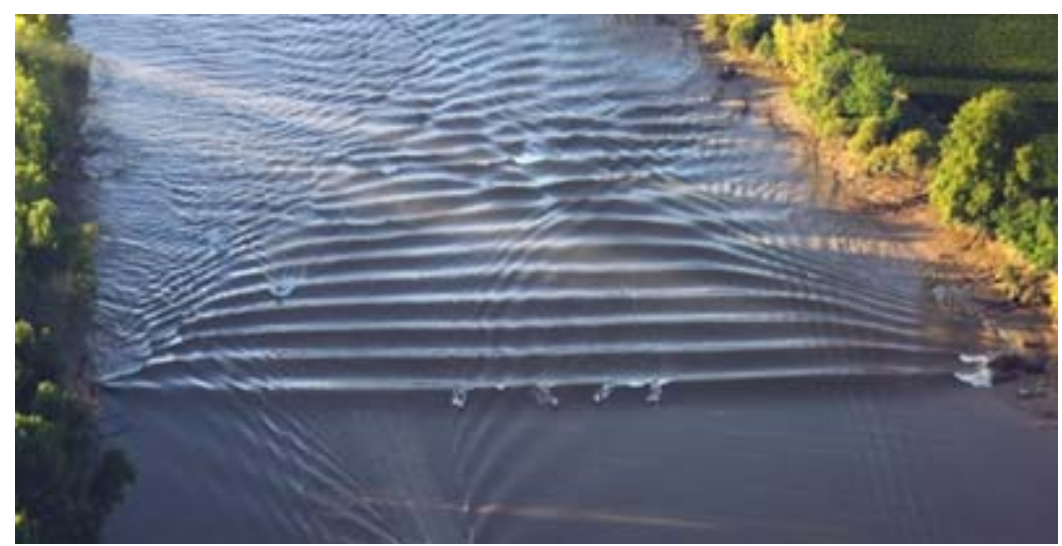

Figure 1. Illustration de la forme caractéristique des RM de forte intensité (i.e. mascarets) dans la Garonne. Photo aérienne réalisée le 10 septembre 2010 à 500m en aval de notre site de mesure de Podensac.

\section{Sites d'étude et méthodes}

Nous avons réalisé nos campagnes de mesures sur deux des principaux systèmes fluvioestuariens français : la Garonne/Gironde et la Seine. Des mesures hydro-sédimentaires intensives ont été réalisées sur la Garonne à Podensac en 2010 (cf. BONNETON et al., 2011 ; 2012a ; 2012b) autour des marées d'équinoxe de printemps, du 24 février au 15 avril (campagne TBG1), et des marées d'équinoxe d'automne, du $1^{\mathrm{er}}$ septembre au 22 octobre (campagne TBG2). Le site de Podensac se situe à $140 \mathrm{~km}$ en amont de l'estuaire, dans une zone où l'onde de marée atteint son amplitude maximale. Des 


\section{XII ${ }^{\text {èmes }}$ Journées Nationales Génie Côtier - Génie Civil \\ Cherbourg, 12-14 juin 2012}

mesures complémentaires ont été réalisées autour des marées d'équinoxe d'automne 2011, à la fois sur la Garonne à Podensac et sur la Seine à Val des Leux qui est situé à $100 \mathrm{~km}$ en amont de l'estuaire.

Dans cet article nous analysons uniquement les mesures d'élévation de la surface libre. Celles-ci ont été obtenues principalement à partir de capteurs de pression (Ocean Sensor System) programmés à une fréquence d'acquisition de $10 \mathrm{~Hz}$. Des mesures acoustiques directes de la surface libre à $2 \mathrm{~Hz}$ ont aussi été réalisées à partir d'un AWAC (Nortek). Une correction non-hydrostatique, basée sur la théorie linéaire des vagues, a été appliquée aux mesures de pression. Cette méthode a été confrontée aux mesures directes de l'AWAC et donne une erreur maximale pour l'amplitude des ondes secondaires les plus cambrées de $10 \%$. Des relevés bathymétriques précis nous ont permis de calculer les profondeurs hydrauliques D et le nombre de Froude F pour les sections de fleuve instrumentés. Le nombre de Froude est déterminé à partir des mesures de hauteurs d'eau à l'avant et à l'arrière du front d'onde moyen et en appliquant la conservation de la masse et de la quantité de mouvement à travers une section variable.

\section{Formation des ressauts de marée}

Les observations sur la Garonne en 2010 sur près de 200 marées ont permis de quantifier pour la première fois l'occurrence des $R M$ dans cet environnement (cf. BONNETON et al. 2012a). Pour la campagne TBG1, réalisée à la fin de l'hiver autour des marées d'équinoxe de printemps, des $R M$ sont observés pour 60 à $64 \%$ des marées. Pour les marées d'équinoxe d'automne (campagne TBG2) on observe, pour le même type de forçage tidal à l'entrée de l'estuaire, des $R M$ plus intenses avec une occurrence de l'ordre de 85 à 90\%. L'incertitude sur la quantification de l'occurrence vient du fait que pour des nombres de Froude faiblement supérieurs à 1, l'identification des ressauts et de leur champ d'ondes secondaires devient difficile (cf. discussion de la figure 3). La différence marquée entre les deux campagnes de mesures est liée à des débits fluviaux très différents. A la fin de l'été on observe une forte amplification de l'onde de marée lors de sa propagation entre l'entrée de l'estuaire et Podensac (cf. BONNETON et al. 2012b). Les forts débits à la fin de l'hiver vont atténuer très fortement ce phénomène et donc être moins favorable à la formation de $R M$.

Des mesures complémentaires ont été réalisées à Podensac en 2011 lors des marées d'équinoxe d'automne (de vive eau à morte eau) pour des débits fluviaux similaires à ceux de TBG2. Des ressauts de marée ont été identifiés pour l'ensemble des 16 marées analysées. La différence d'occurrence des $R M$ entre cette campagne 2011 et TBG2 est due au fait qu'en 2010 le coefficient de marée minimal était de 32 alors qu'en 2011 la marée de morte eau que nous avons analysée était de plus forte amplitude avec un coefficient de 42. La figure 2 présente les ressauts observés en 2011 pour les deux situations extrêmes que sont les marées de vive eau et morte eau. Pour la marée de vive eau (cf. figure 2a) on observe un $R M$ intense (i.e. mascaret), caractérisé par un nombre 
de Froude de 1.24 et des ondes secondaires de fortes amplitudes. La hauteur du premier front d'onde, $\mathrm{H}_{\mathrm{b}}$, atteint $1.57 \mathrm{~m}$ et la période de la première vague est de $2.6 \mathrm{~s}$. La structure du $R M$ présentée sur la figure 2a est représentative des mascarets, avec un champ d'ondes secondaires fortement cambrées et organisé sous forme de groupes d'ondes (modulation à une période de l'ordre de 25s). Le déferlement se produit sur les berges et localement au milieu du fleuve pour les vagues les plus intenses au sein des groupes d'ondes (cf. BONNETON et al., 2012a). Pour la marée de morte eau (cf. figure $2 \mathrm{~b})$ on observe un $R M$ de très faible intensité $(\mathrm{F}=1.04)$ et un champ d'ondes secondaires peu développé
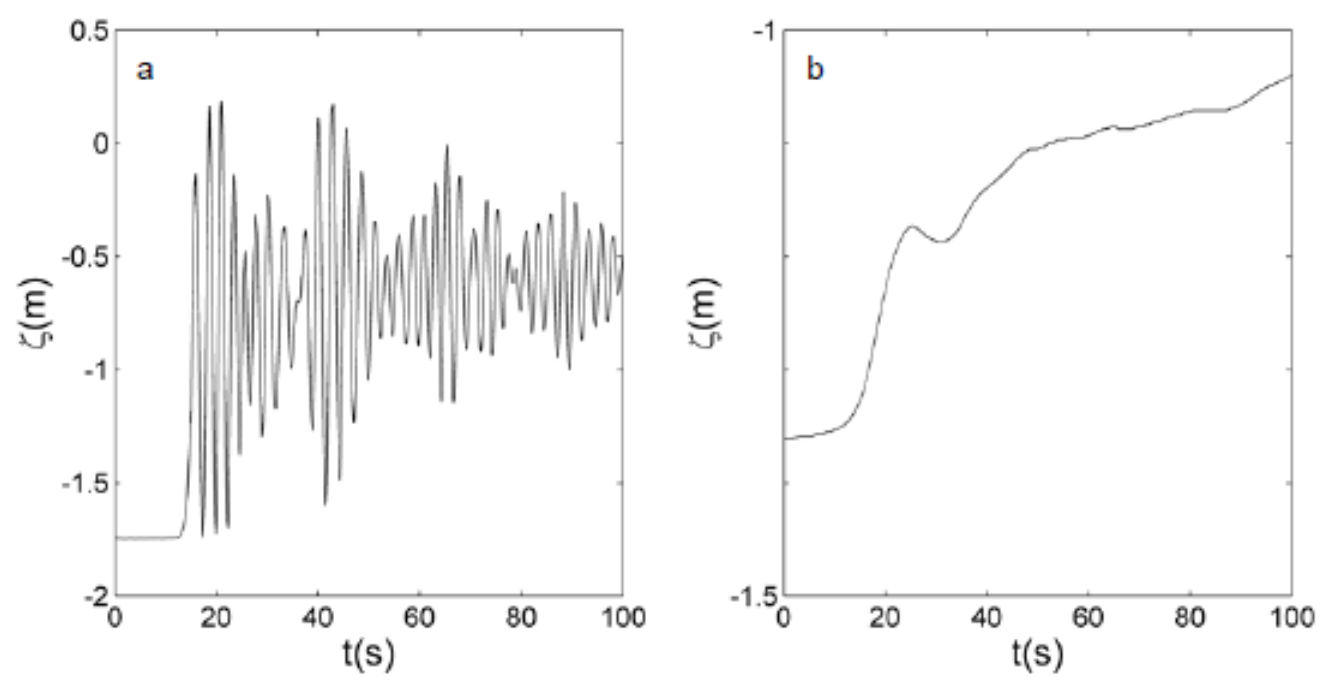

Figure 2. Evolution temporelle de l'élévation du plan d'eau (cote NGF-IGN69) à

Podensac (Garonne) au début de la marée montante. Marées d'équinoxe 2011 : a, marée de vive eau le 31 aout (5 h38' $T U$ ), coef $=111, T_{R L}=6.17 \mathrm{~m}, F=1.24$; $b$, marée de morte eau le 6 septembre (22h49' $T U$ ), coef $=42, T_{R L}=4.27 \mathrm{~m}, F=1.04 . T_{R L}:$ marnage local sur le site de mesure.

$\left(\mathrm{H}_{\mathrm{b}}=0.19 \mathrm{~m}\right)$ avec des oscillations à des périodes beaucoup plus longues que celles des mascarets, de l'ordre de $15 \mathrm{~s}$. Bien que de très faible intensité, ce $R M$ est néanmoins caractérisé par une vitesse d'élévation maximale, $\mathrm{d} \zeta /\left.\mathrm{dt}\right|_{\max }=2.210^{-2} \mathrm{~m} / \mathrm{s}$, d'au moins un ordre de grandeur plus forte que la vitesse d'élévation maximale observée pour les rare cas sans $R M$ lors des marées d'équinoxe d'automne en 2010. Nos résultats montrent que les $R M$ se forment dans la Garonne pour une large majorité de marée et qu'ils peuvent même se produire pour des marées de morte eau. 

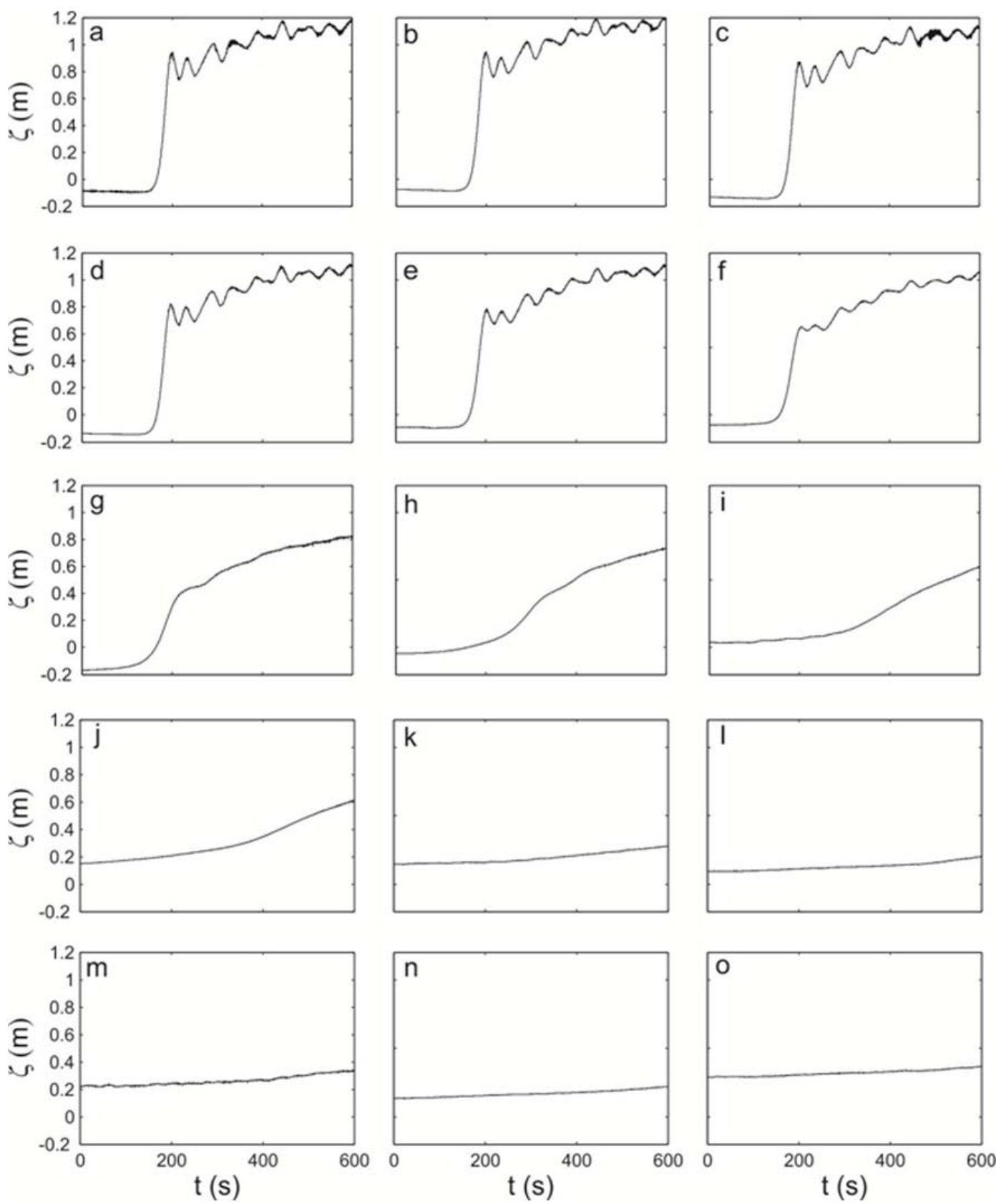

Figure 3. Evolution temporelle de l'élévation du plan d'eau (cote NGF-IGN69) à Val des Leux (Seine) au début de la marée montante. Marées d'équinoxe en 2011 du 31 aout (10 h42' TU) au 7 septembre (16h42' TU). a, coef =111, $T_{R L}=3.68 \mathrm{~m}, F=1.07$; $b$, coef $=108, T_{R L}=3.65 \mathrm{~m}, F=1.07 ; c$, coef $=105, T_{R L}=3.72 \mathrm{~m}, F=1.07 ; d$, coef $=99$,

$T_{R L}=3.72 \mathrm{~m}, F=1.06 ;$ e, coef $=92, T_{R L}=3.61 \mathrm{~m}, F=1.06 ; f$, coef $=85, T_{R L}=3.44 \mathrm{~m}$, $F=1.05 ; g$, coef $=76, T_{R L}=3.40 \mathrm{~m}, 1.04 ; h$, coef $=67, T_{R L}=3.14 \mathrm{~m} ; i$, coef $=59$,

$T_{R L}=3.13 \mathrm{~m} ; j$, coef $=51, T_{R L}=2.75 \mathrm{~m} ; \mathrm{k}$, coef $=45, T_{R L}=2.60 \mathrm{~m} ; l$, coef $=42, T_{R L}=2.41 \mathrm{~m}$; $m$, coef $=42, T_{R L}=2.24 m ; n$, coef $=45, T_{R L}=2.45 m ; o$, coef $=49, T_{R L}=2.35 m . T_{R L}:$ marnage local sur le site de mesure. 
La figure 3 présente l'évolution temporelle de l'élévation du plan d'eau à Val des Leux (Seine), au début de la marée montante, pour les marées d’équinoxe de 2011 comprises entre le 31 août et le 7 septembre. Ces observations, réalisées parallèlement à celles sur la Garonne (cf. figure 2), représentent les premières mesures de $R M$ ondulants dans la Seine. Pour les 6 marées les plus intenses (figures 3a-f) on observe la présence de $R M$ ondulants bien développés caractérisés par des nombres de Froude compris entre 1.05 et 1.07. Pour la marée la plus intense (figure 3a) la hauteur du premier front d'onde est de $1.02 \mathrm{~m}$ et la période T est égale à 34s. Pour les marées de faibles amplitudes (figures 3jo) on observe une évolution progressive de la marée montante, sans présence de $R M$. Les figures 3g-i illustrent la transition progressive entre les $R M$ bien développés et l'absence de $R M$. Sur la figure $3 g$ on observe un $R M$ très faible $(\mathrm{F}=1.04)$ qui devient pratiquement imperceptible lors de la marée suivante (figure $3 \mathrm{~h}$ ).

\section{Transition entre les $R M$ ondulés de faibles amplitudes et les mascarets}

Dans des canaux rectangulaires les caractéristiques des ressauts ondulés non-déferlants évoluent de façon continue en fonction de l'intensité du ressaut. Lorsque le nombre de Froude augmente l'amplitude des ondes secondaires croît et la longueur d'onde, $\lambda$, décroît (ex. : FAVRE, 1935 ; TRESKE, 1994 ; CHANSON, 2011). Cette évolution est assez bien reproduite par la théorie de LEMOINE (1948) comme le montre la figure 4. En milieu naturel les estuaires et les cours d'eau ont généralement des berges en pente douce. Cette propriété à un très fort impact sur la structure des ondes secondaires par rapport à ce qu'on peut observer dans des canaux rectangulaires (cf. BONNETON et al., 2012a). Si la structure du ressaut moyen est pratiquement homogène dans la direction transverse au fleuve, en revanche le champ d'onde secondaire va présenter une très forte variabilité spatiale (cf. figure 1). De plus les caractéristiques du champ d'onde ne vont pas évoluer de façon continue en fonction du nombre de Froude. La figure 4 présente l'évolution de la longueur d'onde de la première vague, adimensionnée par la profondeur hydraulique à l'avant du ressaut, en fonction de F. Pour la Garonne nous ne présentons que des mesures réalisées au voisinage de l'axe du fleuve. Pour $F>F_{c}\left(F_{c} \sim 1.1\right.$ à Podensac), on observe des valeurs en accord avec celles mesurées pour des ressauts ondulés se propageant dans un canal rectangulaire. En revanche lorsque $F<F_{c}$ le mode haute fréquence précédent voit son amplitude décroitre brutalement et il laisse place à des ondes de longueurs d'onde beaucoup plus grandes. Un tel phénomène avait déjà été observé par TRESKE (1994) pour des ressauts en translation se propageant dans un canal trapézoïdal. Lorsque $\mathrm{F}>\mathrm{F}_{\mathrm{c}}$, la cambrure des ondes secondaires est suffisamment intense pour que le $R M$ soit observable visuellement (cf. figure 1) et il est alors dénommé dans le langage courant mascaret. Par contre pour $\mathrm{F}<\mathrm{F}_{\mathrm{c}}$ le $R M$ est de faible cambrure et difficilement observable sans instrumentation hydrodynamique spécifique. Les $R M$ ondulés que nous avons identifiés sur la Seine appartiennent aussi à ce régime 


\section{XII ${ }^{\text {èmes }}$ Journées Nationales Génie Côtier - Génie Civil \\ Cherbourg, 12-14 juin 2012}

"faible cambrure". Ceci explique pourquoi malgré des hauteurs de vagues très significatives $\left(\mathrm{H}_{\mathrm{w}} \sim 1 \mathrm{~m}\right)$ ces $R M$ n'avaient pas été identifiés sur la Seine.

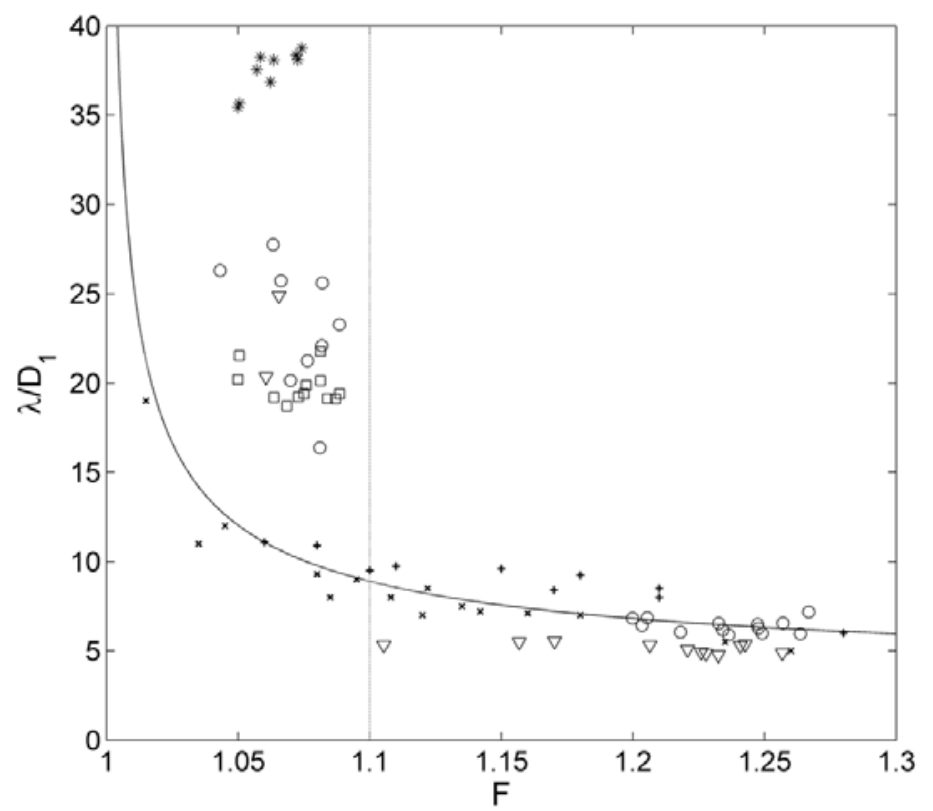

Figure 4. Longueur d'onde adimensionnée en fonction du nombre de Froude F. $\square$, TBG1 ; O, TBG2 ; $\nabla$, Garonne 2011 ; *, Seine 2011 ; x, canal rectangulaire TRESKE (1994) ; +, canal rectangulaire FAVRE (1935); ——, théorie de LEMOINE (1948).

\section{Conclusions}

Nous avons montré que dans un milieu fluvial naturel, avec des berges en pente douce, il existe un changement de régime pour les $R M$ oscillants autour d'un nombre de Froude critique $\mathrm{F}_{\mathrm{c}}$. On observe une brusque augmentation de la cambrure des ondes secondaires lorsque $\mathrm{F}$ dépasse $\mathrm{F}_{\mathrm{c}}$. Ce type de $R M$, énergétique et visuellement observable, correspond au phénomène dénommé mascaret dans le langage courant. Nous avons montré que l'occurrence des $R M$ en milieu fluvio-estuarien était jusqu'à présent fortement sous-estimée (CHANSON, 2011), car le régime "faible cambrure" $\left(\mathrm{F}<\mathrm{F}_{\mathrm{c}}\right)$ n'est pas facilement appréhendable sans mesure hydrodynamique quantitative. Nous avons en particulier montré que dans la Garonne les $R M$ étaient présents pour une large majorité de marées et que par faible débit fluviale ils pouvaient même se développer par marée de morte eau. Nous avons pu aussi identifier des $R M$ ondulés de "faible cambrure" dans la Seine et présenter les premières mesures quantitatives de ce phénomène. Cette réévaluation de l'occurrence des ressauts de marée, en particulier dans des environnements comme la Seine où ils semblaient, suite à des aménagements, avoir en partie disparus, ouvre de nouvelles perspectives sur le rôle qu'ils jouent dans les écosystèmes fluvio-estuariens. 


\section{Remerciements :}

Ce travail a été réalisé dans le cadre du projet MASCARET (Région Aquitaine), avec des financements complémentaires de l’Université de Bordeaux 1. Nous remercions l'ensemble des personnes qui ont été impliquées dans ces campagnes de mesures et tout particulièrement G. Detandt, G. Oggian, N. Pochon (EPOC) et F. Pradalier (CKBN). Les VNF de Cadillac nous ont été d'un très grand soutien pour le mouillage des instruments dans la Garonne, ainsi que Y. Lavigne pour les photos aériennes. Nous remercions P. Tournier et C. Guillon (Grand Port Maritime de Rouen) et J-P. Lemoine (GIP Seine Aval) pour leur aide lors de la campagne de mesures sur la Seine.

\section{Références bibliographiques}

BONNETON N., BONNETON P, PARISOT J-P., DETANDT G., SOTTOLICHIO A., CRAPOULET A. (2011). Structure verticale des courants associés à la propagation de la marée dans la Garonne - Impact du mascaret. $20{ }^{\text {ème }}$ Congrès Français de Mécanique, $6 \mathrm{p}$.

BONNETON P., VAN DE LOOCK J., PARISOT J-P., BONNETON N., SOTTOLICHIO A., DETANDT G., CASTELLE B., MARIEU V., POCHON N., (2012a). On the occurrence of tidal bores -The Garonne River case. Journal of Coastal Research, SI 64, in press.

BONNETON P, PARISOT J-P., BONNETON N., SOTTOLICHIO A., CASTELLE B., MARIEU V., POCHON N., VAN DE LOOCK J. (2012b). Large amplitude undular tidal bore propagation in the Garonne River, France. ISOPE , in press.

CHANSON H. (2011). Tidal bores, aegir, eagre, mascaret, pororoca. Theory and observations. World Scientific Publishing Co. Pte. Ltd.

FAVRE H. (1935). Etude théorique et expérimentale des ondes de translation dans les canaux découverts. Dunod, Paris.

LEMOINE R. (1948). Sur les ondes positives de translation dans les canaux et sur le ressaut ondulé de faible amplitude. Houille Blanche, Vol. 2, pp 183-185.

SIMPSON J.H., FISHER N.R., WILES P. (2004). Reynolds stress and TKE production in an estuary with a tidal bore. Estuarine, Coastal and Shelf Science, Vol. 60, pp 619-627. doi:10.1016/j.ecss.2004.03.006

TRESKE A. (1994). Undular bore (Favre-waves) in open channels - Experimental studies. Journal of Hydraulic Research, Vol. 32, n 3, pp 355-370.

WOLANSKI E, WILLIAMS D, SPAGNOL S., CHANSON H. (2004). Undular tidal bore dynamics in the Daly Estuary, Northern Australia. Estuarine Coastal and Shelf Science, Vol. 60 (4), pp 629-636. doi:10.1016/j.ecss.2004.03.001 